\title{
ON PROPERTIES OF CERTAIN ANALYTIC MULTIPLIER TRANSFORM OF COMPLEX ORDER
}

\author{
DEBORAH OLUFUNMILAYO MAKINDE ${ }^{1, *}$, SHAHRAM NAJAFZADEH ${ }^{2}$ \\ ${ }^{1}$ Department of Mathematics, Obafemi Awolowo University, Ile-Ife, Nigeria \\ ${ }^{2}$ Department of Mathematics, Payame Noor University, P. O. Box: 19395-3697, Tehran, Iran \\ *Corresponding author: funmideb@yahoo.com
}

\begin{abstract}
The focus of this paper is to investigate the subclasses $S^{*} C(\gamma, \mu, \alpha, \lambda ; b), T S^{*} C(\gamma, \mu, \alpha, \lambda ; b)=$ $T \cap S^{*} C(\gamma, \mu, \alpha, \lambda ; b)$ and obtain the coefficient bounds as well as establishing its relationship with certain existing results in the literature.
\end{abstract}

\section{Introduction}

Let $A$ be the class of normalized analytic functions $f$ in the open unit disc $U=\{z \in C:|z|<1\}$ with $f(0)=f^{\prime}(0)=0$ and of the form

$$
f(z)=z+\sum_{n=2}^{\infty} a_{n} z^{n}, \quad a_{n} \in C,
$$

and $S$ the class of all functions in $A$ that are univalent in $U$. Also, the subclass of functions in $A$ that are of the form

$$
f(z)=z-\sum_{n=2}^{\infty} a_{n} z^{n}, \quad a_{n} \geq 0
$$

is denoted by $T$ and the subclasses $S^{*}(\alpha), \quad C(\gamma)$ are given respectively by

$$
S^{*}(\alpha)=\left\{f \in S: \operatorname{Re}\left(\frac{z f^{\prime}(z)}{f(z)}\right)>\gamma z \in U\right\}
$$

Received 2019-07-05; accepted 2019-08-12; published 2019-11-01.

2010 Mathematics Subject Classification. 30C45.

Key words and phrases. analyticity; univalent; linear transformation; coefficient bounds.

(C)2019 Authors retain the copyrights of their papers, and all open access articles are distributed under the terms of the Creative Commons Attribution License. 


$$
C(\alpha)=\left\{f \in S: \operatorname{Re}\left(1+\frac{z f^{\prime \prime}(z)}{f^{\prime}(z)}\right)>\gamma z \in U, \geq \gamma<1\right\} .
$$

Moreover, the class $T S^{*}(\gamma)$ denoted by $T \cap S^{*}(\gamma)$ which is the subclass of function $f \in T$ such that $f$ is starlike of order $\gamma$ and respectively, $T C(\gamma)$ is the class of function $f \in T$ such that $f$ is convex of order $\gamma$. An interesting unification of the classes $S^{*}(\alpha)$ and $C(\gamma)$ denoted by $S^{*} C(\gamma, \beta)$ which satisfies the condition

$$
\operatorname{Re}\left\{\frac{z f^{\prime}(z)+\beta z^{2} f^{\prime}(z)}{\beta z f^{\prime}(z)+(1-\beta) f(z)}\right\}>\gamma \quad 0 \geq \gamma<1, z \in U
$$

has been extensively studied by different researchers, for example, see [6] and $[1,2,3]$. The special cases for $\beta=0,1$ are given by $S^{*}(\gamma)$ and $C((\gamma))$ respectively.

Furthermore, the class $T S^{*} C(\gamma, \beta)$ which is the subclass of function $f \in T$ such that $f$ belongs the class $S^{*} C(\gamma, \beta)$, was studied by Altintas et al. and other researchers. For details see [ $\left.3,5,6\right]$.

Using the unification in (5), Nizami Mustafa [6] introduced and investigated the class $S^{*} C(\gamma, \beta ; \tau)$ and $T S^{*} C(\gamma, \beta ; \tau), 0 \leq \alpha<1 ; \beta \in[0,1] ; \tau \in C$ which he defined as follows

A function $f \in S$ given by (1.1) is said to belong to the class $S^{*} C(\gamma, \beta ; \tau)$ if the following condition is satisfied

$$
\operatorname{Re}\left\{1+\frac{1}{\tau}\left[\frac{z f^{\prime}(z)+\beta z^{2} f^{\prime}(z)}{\beta z f^{\prime}(z)+(1-\beta) f(z)}-1\right]\right\}>\gamma \quad 0 \geq \gamma<1 ; \beta \in[0,1] ; \tau \in C-\{0\}, z \in U .
$$

Meanwhile, the author in [4] defined a linear transformation $D_{\alpha, \lambda}^{m} f$ by

$$
D_{\alpha, \lambda}^{m} f(z)=z+\sum_{n=2}^{\infty} \alpha\left(\frac{1+\lambda(n+\alpha-2)}{1+\lambda(\alpha-1)}\right)^{m} a_{n} z^{n}, \quad 0 \leq \lambda \leq 1 ; \alpha \geq 1 ; m \in \mathbb{N} \cup 0
$$

Motivated by the work of Mustafa in [6], we study the effect of the application of the linear operator $D_{\alpha, \lambda}^{m} f$ on the unification of the classes of the functions $S^{*} C(\gamma, \beta ; \tau)$.

Now, we define the class $S^{*} C(\gamma, \alpha, \lambda ; b)$ to be class of functions $f \in S$ which satisfies the condition

$\operatorname{Re}\left\{1+\frac{1}{b}\left[\frac{z\left(D_{\alpha, \lambda}^{m} f\right)^{\prime}(z)+\mu z^{2}\left(D_{\alpha, \lambda}^{m} f\right)^{\prime \prime}(z)}{\mu z\left(D_{\alpha, \lambda}^{m} f\right)^{\prime}(z)+(1-\mu)\left(D_{\alpha, \lambda}^{m} f\right)(z)}-1\right]\right\}>\gamma, 0 \geq \gamma<1, z \in U ; 0 \leq \lambda, \mu \leq 1 ; \alpha \geq 1 ; m \in \mathbb{N} \cup 0$

Also, we denote by $D_{T}$ the subclass of the class of functions in (7) which is of the form

$$
D_{\alpha, \lambda}^{m} f(z)=z-\sum_{n=2}^{\infty} \alpha\left(\frac{1+\lambda(n+\alpha-2)}{1+\lambda(\alpha-1)}\right)^{m} a_{n} z^{n}, \quad 0 \leq \lambda, \mu \leq 1 ; \alpha \geq 1 ; m \in \mathbb{N} \cup 0
$$

and denote by $T S^{*} C(\gamma, \mu, \alpha, \lambda ; b)=T \cap S^{*} C(\gamma, \mu, \alpha, \lambda ; b)$ which is the class of functions $f$ in (1.9) such that $f$ belong to the class $S^{*} C(\gamma, \mu, \alpha, \lambda ; b)=T \cap S^{*} C(\gamma, \mu, \alpha, \lambda ; b)$.

In this paper, we investigate the subclasses $S^{*} C(\gamma, \mu, \alpha, \lambda ; b)$ and $T S^{*} C(\gamma, \mu, \alpha, \lambda ; b)=T \cap S^{*} C(\gamma, \mu, \alpha, \lambda ; b)$ 
2. Coeffiecient Bounds for the Classes $S^{*} C_{\alpha}^{\lambda}(\gamma, \mu ; b)$ And $T S^{*} C_{\alpha}^{\lambda}(\gamma, \mu ; b)$

Theorem 2.1. Let $f$ be as defined in (1.1). Then the function $D_{\alpha, \lambda}^{m} f$ belongs to the class $S^{*} C(\gamma, \mu, \alpha, \lambda ; b)$, $0 \geq \gamma<1, z \in U ; 0 \leq \lambda, \mu \leq 1 ; \alpha \geq 1 ; m \in \mathbb{N} \cup 0$

if

$$
\sum_{n=2}^{\infty}\left[\alpha\left(\frac{1+\lambda(n+\alpha-2)}{1+\lambda(\alpha-1)}\right)^{m}[1+\mu(n-1)][n+|b|(1-\gamma)-1]\right]\left|a_{n}\right| \leq|b|(1-\gamma)
$$

The result is sharp for the function

$$
D_{\alpha, \lambda}^{m} f(z)=z+\frac{|b|(1-\gamma)(1+\lambda(\alpha-1))^{m}}{\alpha[1+\mu(n-1)][n+|b|(1-\gamma)](1+\lambda(n+\alpha-2))^{m}} z^{n} \quad n \geq 2
$$

Proof. By (1.8), $f$ belong to the class $S^{*} C(\gamma, \mu, \alpha, \lambda ; b)$ if

$$
\operatorname{Re}\left\{1+\frac{1}{b}\left[\frac{z\left(D_{\alpha, \lambda}^{m} f\right)^{\prime}(z)+\mu z^{2}\left(D_{\alpha, \lambda}^{m} f\right)^{\prime \prime}(z)}{\mu z\left(D_{\alpha, \lambda}^{m} f\right)^{\prime}(z)+(1-\mu)\left(D_{\alpha, \lambda}^{m} f\right)(z)}-1\right]\right\}>\gamma
$$

It suffices to show that:

$$
\left|\frac{1}{b}\left[\frac{z\left(D_{\alpha, \lambda}^{m} f\right)^{\prime}(z)+\mu z^{2}\left(D_{\alpha, \lambda}^{m} f\right)^{\prime \prime}(z)}{\mu z\left(D_{\alpha, \lambda}^{m} f\right)^{\prime}(z)+(1-\mu)\left(D_{\alpha, \lambda}^{m} f\right)(z)}-1\right]\right|<1-\gamma
$$

Simple computation in (2.1), using (1.7), we have:

$$
\begin{gathered}
\left|\frac{1}{b}\left[\frac{z\left(D_{\alpha, \lambda}^{m} f\right)^{\prime}(z)+\mu z^{2}\left(D_{\alpha, \lambda}^{m} f\right)^{\prime \prime}(z)}{\mu z\left(D_{\alpha, \lambda}^{m} f\right)^{\prime}(z)+(1-\mu)\left(D_{\alpha, \lambda}^{m} f\right)(z)}-1\right]\right| \\
=\left|\frac{1}{b}\left[\frac{z+\sum_{n=2}^{\infty} n \alpha\left(\frac{1+\lambda(n+\alpha-2)}{1+\lambda(\alpha-1)}\right)^{m} a_{n} z^{n}+\mu \sum_{n=2}^{\infty} n(n-1) \alpha\left(\frac{1+\lambda(n+\alpha-2)}{1+\lambda(\alpha-1)}\right)^{m} a_{n} z^{n}}{\mu z+\sum_{n=2}^{\infty} \mu n \alpha\left(\frac{1+\lambda(n+\alpha-2)}{1+\lambda(\alpha-1)}\right)^{m} a_{n} z^{n}+(1-\mu)\left(z+\sum_{n=2}^{\infty} \alpha\left(\frac{1+\lambda(n+\alpha-2)}{1+\lambda(\alpha-1)}\right)^{m} a_{n} z^{n}\right)}-1\right]\right| \\
=\left|\frac{1}{b}\left[\frac{z+\sum_{n=2}^{\infty} n \alpha[1+\mu(n-1)]\left(\frac{1+\lambda(n+\alpha-2)}{1+\lambda(\alpha-1)}\right)^{m} a_{n} z^{n}}{z+\sum_{n=2}^{\infty} \alpha(1+\mu(n-1))\left(\frac{1+\lambda(n+\alpha-2)}{1+\lambda(\alpha-1)}\right)^{m} a_{n} z^{n}}-1\right]\right| \\
\leq \frac{1}{b}\left[\frac{\sum_{n=2}^{\infty} \alpha(n-1)[1+\mu(n-1)]\left(\frac{1+\lambda(n+\alpha-2)}{1+\lambda(\alpha-1)}\right)^{m}\left|a_{n}\right|}{1-\sum_{n=2}^{\infty} \alpha(1+\mu(n-1))\left(\frac{1+\lambda(n+\alpha-2)}{1+\lambda(\alpha-1)}\right)^{m}\left|a_{n}\right|}\right]
\end{gathered}
$$

which is bounded by $1-\gamma$ if

$\sum_{n=2}^{\infty} \alpha(n-1)[1+\mu(n-1)]\left(\frac{1+\lambda(n+\alpha-2)}{1+\lambda(\alpha-1)}\right)^{m}\left|a_{n}\right| \leq|b|(1-\gamma) 1-\sum_{n=2}^{\infty} \alpha(1+\mu(n-1))\left(\frac{1+\lambda(n+\alpha-2)}{1+\lambda(\alpha-1)}\right)^{m}\left|a_{n}\right|$ which is equivalent to

$\sum_{n=2}^{\infty}\left[\alpha(n-1)[1+\mu(n-1)]\left(\frac{1+\lambda(n+\alpha-2)}{1+\lambda(\alpha-1)}\right)^{m}+\alpha|b|(1-\gamma)(1+\mu(n-1))\left(\frac{1+\lambda(n+\alpha-2)}{1+\lambda(\alpha-1)}\right)^{m}\right]\left|a_{n}\right| \leq|b|(1-\gamma)$ Which implies that

$$
\sum_{n=2}^{\infty}\left[\alpha\left(\frac{1+\lambda(n+\alpha-2)}{1+\lambda(\alpha-1)}\right)^{m}[1+\mu(n-1)][n+|b|(1-\gamma)-1]\right]\left|a_{n}\right| \leq|b|(1-\gamma)
$$


Thus, (2.1) is satisfied if (2.2) is satisfied.

Corollary 2.1. Let $f$ be as defined in (1) and the function $D_{\alpha, \lambda}^{m} f$ belongs to the class $S^{*} C(\gamma, \mu, \alpha, \lambda ; b)$, $0 \geq \gamma<1, z \in U ; 0 \leq \lambda, \mu \leq 1 ; \alpha \geq 1 ; m \in \mathbb{N} \cup 0$. Then

$$
\left|a_{n}\right| \leq \frac{|b|(1-\gamma)(1+\lambda(\alpha-1))^{m}}{\alpha[1+\mu(n-1)][n+|b|(1-\gamma)-1](1+\lambda(n+\alpha-2))^{m}}
$$

Corollary 2.2. Let $f$ be as defined in (1.1). Then the function $D_{\alpha, \lambda}^{m} f$ belongs to the class $S^{*} C(\gamma, \mu, 1, \lambda, m ; b)$, $0 \geq \gamma<1, z \in U ; 0 \leq \lambda, \mu \leq 1 ; m \in \mathbb{N} \cup 0$ if

$$
\sum_{n=2}^{\infty}\left[(1+\lambda(n-1))^{m}[1+\mu(n-1)][n+|b|(1-\gamma)-1]\right]\left|a_{n}\right| \leq|b|(1-\gamma)
$$

The result is sharp for the function

$$
D_{\alpha, \lambda}^{m} f(z)=z+\frac{|b|(1-\gamma)}{[1+\mu(n-1)][n+|b|(1-\gamma)-1](1+\lambda(n-1))^{m}} z^{n}, \quad n \geq 2
$$

Corollary 2.3. Let $f$ be as defined in (1.1). Then the function $D_{\alpha, \lambda}^{m} f$ belongs to the class $S^{*} C(\gamma, \mu, 1, \lambda, 1 ; b)$, $0 \geq \gamma<1, z \in U ; 0 \leq \lambda, \mu \leq 1 ; m \in \mathbb{N} \cup 0$ if

$$
\sum_{n=2}^{\infty}[(1+\lambda(n-1))[1+\mu(n-1)][n+|b|(1-\gamma)-1]]\left|a_{n}\right| \leq|b|(1-\gamma)
$$

The result is sharp for the function

$$
D_{\alpha, \lambda}^{m} f(z)=z+\frac{|b|(1-\gamma)}{[1+\mu(n-1)][n+|b|(1-\gamma)-1](1+\lambda(n-1))} z^{n}, \quad n \geq 2
$$

Corollary 2.4. Let $f$ be as defined in (1.1). Then the function $D_{\alpha, \lambda}^{m} f$ belongs to the class $S^{*} C(\gamma, \mu, 1,1,1 ; b)$, $0 \geq \gamma<1, z \in U ; 0 \leq \lambda, \mu \leq 1 ; m \in \mathbb{N} \cup 0$ if

$$
\sum_{n=2}^{\infty}[n[1+\mu(n-1)][n+|b|(1-\gamma)-1]]\left|a_{n}\right| \leq|b|(1-\gamma)
$$

The result is sharp for the function

$$
D_{\alpha, \lambda}^{m} f(z)=z+\frac{|b|(1-\gamma)}{n[1+\mu(n-1)][n+|b|(1-\gamma)-1]} z^{n}, \quad n \geq 2
$$

Corollary 2.5. Let $f$ be as defined in (1.1). Then the function $D_{\alpha, \lambda}^{m} f$ belongs to the class $S^{*} C(\gamma, \mu, 1,0,1 ; b)$, $0 \geq \gamma<1, z \in U ; 0 \leq \lambda, \mu \leq 1 ; m \in \mathbb{N} \cup 0$ if

$$
\sum_{n=2}^{\infty}[[1+\mu(n-1)][n+|b|(1-\gamma)-1]]\left|a_{n}\right| \leq|b|(1-\gamma)
$$

The result is sharp for the function

$$
D_{\alpha, \lambda}^{m} f(z)=z+\frac{|b|(1-\gamma)}{[1+\mu(n-1)][n+|b|(1-\gamma)-]} z^{n}, \quad n \geq 2
$$

This result agrees with the Theorem 2.1 in [6]. 
Corollary 2.6. Let $f$ be as defined in (1.1). Then the function $D_{\alpha, \lambda}^{m} f$ belongs to the class $S^{*} C(\gamma, 0,1, \lambda, 0 ; 1)$, $0 \geq \gamma<1, z \in U ; 0 \leq \lambda, \mu \leq 1 ; m \in \mathbb{N} \cup 0$ if

$$
\sum_{n=2}^{\infty}[[1+\mu(n-1)][n-\gamma]]\left|a_{n}\right| \leq 1-\gamma
$$

The result is sharp for the function

$$
D_{\alpha, \lambda}^{m} f(z)=z+\frac{1-\gamma}{[1+\mu(n-1)][n-\gamma]} z^{n}, \quad n \geq 2
$$

This result agrees with the Corollary 2.1 in [6].

Corollary 2.7. Let $f$ be as defined in (1.1). Then the function $D_{\alpha, \lambda}^{m} f$ belongs to the class $S^{*} C(\gamma, \mu, 1, \lambda, 0 ; 1)$, $0 \geq \gamma<1, z \in U ; 0 \leq \lambda, \mu \leq 1 ; m \in \mathbb{N} \cup 0$ if

$$
\sum_{n=2}^{\infty}(n-\gamma)\left|a_{n}\right| \leq 1-\gamma
$$

The result is sharp for the function

$$
D_{\alpha, \lambda}^{m} f(z)=z+\frac{1-\gamma}{n-\gamma} z^{n}, \quad n \geq 2
$$

This result agrees with the Corollary 2.2 in [6].

Theorem 2.2. Let $f \in D_{T}$. Then the function $D_{\alpha, \lambda}^{m} f$ belongs to the class $D_{T} S^{*} C(\gamma, \mu, \alpha, \lambda ; b)$, $0 \geq \gamma<1, z \in U ; 0 \leq \lambda, \mu \leq 1 ; \alpha \geq 1 ; m \in \mathbb{N} \cup 0$ if and only if

$$
\sum_{n=2}^{\infty} \alpha(n-1)[1+\mu(n-1)][n+b(1-\gamma)]\left(\frac{x}{y}\right)^{m}\left|a_{n}\right| \leq|b|(1-\gamma)
$$

Proof. We shall prove only the necessity part of the Theorem as the sufficiency proof is similar to the proof of Theorem 1.

Let $f$ be as defined in (1.1) and $D_{\alpha, \lambda}^{m} f$ belongs to the class $T S^{*} C(\gamma, \mu, \alpha, \lambda ; b), \quad 0 \geq \gamma<1, z \in U ; 0 \leq$ $\lambda, \mu \leq 1 ; \alpha \geq 1 ; m \in \mathbb{N} \cup 0 ; b \in \mathbb{R}-\{0\}$, we have

$$
\operatorname{Re}\left\{1+\frac{1}{b}\left[\frac{z\left(D_{\alpha, \lambda}^{m} f\right)^{\prime}(z)+\mu z^{2}\left(D_{\alpha, \lambda}^{m} f\right)^{\prime \prime}(z)}{\mu z\left(D_{\alpha, \lambda}^{m} f\right)^{\prime}(z)+(1-\mu)\left(D_{\alpha, \lambda}^{m} f\right)(z)}-1\right]\right\}>\gamma
$$

Using (1.7) in (2.9) and by algebraic simplification, we have

$$
\operatorname{Re}\left\{\frac{-\sum_{n=2}^{\infty} \alpha(n-1)[1+\mu(n-1)]\left(\frac{1+\lambda(n+\alpha-2)}{1+\lambda(\alpha-1)}\right)^{m} a_{n} z^{n}}{b\left\{z-\sum_{n=2}^{\infty} \alpha(1+\mu(n-1))\left(\frac{1+\lambda(n+\alpha-2)}{1+\lambda(\alpha-1)}\right)^{m} a_{n} z^{n}\right\}}\right\} \geq \gamma-1
$$

Choosing $z$ to be real and $z \longrightarrow 1$, we have

$$
\frac{-\sum_{n=2}^{\infty} \alpha(n-1)[1+\mu(n-1)]\left(\frac{1+\lambda(n+\alpha-2)}{1+\lambda(\alpha-1)}\right)^{m} a_{n}}{b\left\{1-\sum_{n=2}^{\infty} \alpha(1+\mu(n-1))\left(\frac{1+\lambda(n+\alpha-2)}{1+\lambda(\alpha-1)}\right)^{m} a_{n}\right\}} \geq \gamma-1
$$


$b \in \mathbb{R}-\{0\}$ implies that $b$ could be greater or less than zero.

Let $b>0$ in (19), we have

$$
-\sum_{n=2}^{\infty} \alpha(n-1)[1+\mu(n-1)]\left(\frac{x}{y}\right)^{m} a_{n} \geq(\gamma-1) b\left\{1-\sum_{n=2}^{\infty} \alpha(1+\mu(n-1))\left(\frac{x}{y}\right)^{m} a_{n}\right\}
$$

where $x=1+\lambda(n+\alpha-2)$ and $y=1+\lambda(\alpha-1)$ From (20), we have

$$
\sum_{n=2}^{\infty} \alpha(n-1)[1+\mu(n-1)][n+b(1-\gamma)]\left(\frac{x}{y}\right)^{m}\left|a_{n}\right| \leq b(1-\gamma)
$$

Now suppose $b<0$, which implies that $b=-|b|$ and substituting $b=-|b|$ in (19), we have

$$
\begin{gathered}
\frac{\sum_{n=2}^{\infty} \alpha(n-1)[1+\mu(n-1)]\left(\frac{x}{y}\right)^{m} a_{n}}{|b|\left\{1-\sum_{n=2}^{\infty} \alpha(1+\mu(n-1))\left(\frac{x}{y}\right)^{m} a_{n}\right\}} \geq \\
\sum_{n=2}^{\infty} \alpha(n-1)[1+\mu(n-1)]\left(\frac{x}{y}\right)^{m}\left|a_{n}\right| \geq(\gamma-1)|b|\left\{1-\sum_{n=2}^{\infty} \alpha(1+\mu(n-1))\left(\frac{x}{y}\right)^{m} a_{n}\right\} \text { which implies } \\
\sum_{n=2}^{\infty} \alpha(n-1)[1+\mu(n-1)][n+b(1-\gamma)]\left(\frac{1+\lambda(n+\alpha-2)}{1+\lambda(\alpha-1)}\right)^{m}\left|a_{n}\right| \geq-b(1-\gamma)
\end{gathered}
$$

From (21) and (23), the proof of the necessity is completed.

\section{REFERENCES}

[1] Altintas, O., On a subclass of certain starlike functions with negative coefficient. Math. Japon., 36 (1991), 489-495.

[2] Altintas, O., Irmak, H. and Srivastava, H. M., Fractional calculus and certain starlke functions with negative coefficients. Comput. Math. Appl., 30 (1995), No. 2, 9-16.

[3] Altintas, O., Özkan, Ö. and Srivastava, H. M., Neighborhoods of a Certain Family of Multivalent Functions with Negative Coefficients. Comput. Math. Appl., 47 (2004), 1667- 1672.

[4] Makinde, D.O., A new multiplier differential operator. Adv. Math., Sci. J., 7 (2018), no.2, 109 -114.

[5] Irmak, H., Lee, S. H. and Cho, N. E., Some multivalently starlike functions with negative coefficients and their subclasses defined by using a differential operator. Kyungpook Math. J., 37 (1997), 43-51.

[6] Mustafa, N., The various properties of certain subclasses of analytic functions of complex order. arXiv:1704.04980 [math.CV], 2017. 